CERN-PPE/94-117

30 March 1994

\title{
THE JETSET BARREL DRIFT-TUBE ('STRAW') CHAMBER
}

\author{
N.H. Hamann ${ }^{\dagger)}$, J. Dittmayer, A. Klett, E. Rössle, M. Tscheulin, H.-J. Urban, \\ H. Wirth, H. Zipse \\ Fakultät für Physik, Universität Freiburg, D-79104 Freiburg, Germany \\ M.J. Price, E. David, R. Harfield, R. Jones, D. Lacroix and C. Rivoiron \\ CERN, PPE Division, CH-1211 Geneva 23, Switzerland
}

\begin{abstract}
A charged-particle tracking chamber consisting of about 1400 individual drift tubes ('straws') has been designed and built for the experiment PS202 (JETSET), installed in the antiproton storage ring LEAR at CERN. The design considerations, the development of several new techniques, and the construction of the detector are described. A complex system of front-end electronics, specially developed for the chamber, provides drift-time as well as charge-division measurements. Calibration procedures for obtaining three-dimensional space information for charged-particle tracks are described. The performance of the chamber achieved in the actual experiment is discussed.
\end{abstract}

(Submitted to Nuclear Instruments and Methods A)

†) deceased. 
For several years, drift chambers made of individual thin-walled drift tubes have been operated in experimental high-energy particle and nuclear physics [1]. Such detectors were successfully built and commissioned for a number of experiments: HRS [2], MAC [3], TPC [4] and MARK II [5] at SLAC-PEP, MARK III [6] at SLAC-SPEAR, KEDR [7] at Novosibirsk VEPP-4M, AMY [8] at KEK-TRISTAN, E760 [9] at the FNAL antiproton accumulator, and CDF [10] at the FNAL proton-antiproton collider. In most applications the drift tubes consisted of aluminized or Al-laminated Mylar (hence the appellation 'straws'), but extruded aluminium-alloy or stainless-steel tubes have also been employed. The chambers were operated in the proportional or in the limited-streamer mode, and the gas pressures used were between 1 and 4 atmospheres. Typical transverse and longitudinal resolutions, obtained from drift-time and charge-division measurements, respectively, were $\sigma_{r} \approx 100 \mu \mathrm{m}$ and $\sigma_{z} \approx 0.01 \mathrm{~L}$ for a single wire of length $L$. Small-cell drift tubes have also been chosen as the main elements of large central chambers for charged-particle tracking in experiments at future quark-flavour factories [11] or high-luminosity hadron colliders [12].

The basic principle of a straw drift tube is that of a single-wire proportional chamber. The radial drift-field geometry between the anode wire and the cylindrical cathode surface is well suited to detect, for each track passing through, the first electron arriving at the wire. Multiple tracks in the same straw, however, can cause overlapping signals and thus a certain pile-up effect. Compared to standard planar or cylindrical drift chambers, straws offer the following important advantages:

- Individual straws are cheap and easy to build.

- The modular structure of a straw chamber allows the accommodation of very special geometries imposed by the experimental environment.

- A broken sense wire causes only a small region in the overall detector to be insensitive. This is important in situations where access to the chamber for repair is restricted or even impossible.

- Neighbouring straws are shielded by means of their cathodes, which minimizes crosstalk.

- Transverse $(\perp)$ and longitudinal $(\|)$ space information is easily obtained, whereas the capability of $d E / d x$ determination seems to be less obvious.

- Electrostatic field distortions due to a decentred anode wire only slightly affect the drift time.

- Straws can be made self-supporting; therefore the material needed for mechanical and electrical stability can be minimized.

Most previous straw chambers have been built for 'colliding-beam' experiments in high-energy $\mathrm{e}^{+} \mathrm{e}^{-}$or $\bar{p}$ p storage rings with the implication that particle distributions and kinematics exhibit a certain forward-backward symmetry. It is typical for the barrel-type chambers employed that the straws are kept straight and their anode wires centred by stretching the tubes between massive end plates. In such a way multilayers inclined by small angles can also be implemented for spatial location of the particle tracks (the socalled small-angle stereo technique). An alternative method of keeping straws straight is to glue them side by side to give rigidity to the overall assembly without the need for thick end plates. In some cases, thick aluminium cathodes have been chosen to reduce etching effects caused by sparks in high-radiation environments. The lifetime of the chamber is prolonged and, moreover, the rigidity of the individual tube is improved. Considerable care has been taken concerning the straw-chamber alignment, where the applicability of laser methods is limited owing to the non-transparent tube walls. 
Here we describe the barrel straw chamber that has been developed for experiment PS202 (JETSET) at the CERN Low-Energy Antiproton Ring (LEAR). The entire charged-particle tracking in this experiment relies on drift tubes, where the forward and barrel straw chambers have very similar basic designs and are identically operated. The JETSET barrel straw chamber differs in several aspects from previous designs.

- The wire tension is fully supported by the straws themselves.

- Each straw delivers both drift-time and charge-division information; hence it provides direct three-dimensional space information.

- The chamber is employed in an internal experiment. Small-angle tracks leave the barrel through a thin end-plate assembly and must be further followed in a 'forward' chamber, where the wires are oriented perpendicular to the beam direction.

In section 2 we discuss some considerations that lead us to the particular design chosen. Construction of the full detector is described in section 3. In section 4 we give details of the front-end electronics developed for the chamber. Calibration procedures and the performance achieved in the experiment are described in section 5 .

\section{$2 \quad$ Principal considerations}

\subsection{The JETSET experiment}

The JETSET experiment $[13,14]$ at LEAR studies rare proton-antiproton annihilation channels, which are of special interest in hadron spectroscopy. Examples are $\bar{p} p \rightarrow \phi \phi$ and $\overline{\mathrm{p}} \mathrm{p} \rightarrow \mathrm{K}_{\mathrm{S}} \mathrm{K}_{\mathrm{S}}$, where the detected decay channels are $\phi \rightarrow \mathrm{K}^{+} \mathrm{K}^{-}$and $\mathrm{K}_{\mathrm{S}} \rightarrow \pi^{+} \pi^{-}$, respectively. A scheme of the experimental arrangement is shown in fig. 1 . The experiment is installed in the LEAR machine and uses an internal hydrogen-cluster jet target. The interaction of the circulating unbunched antiproton beam with the hydrogen jet can provide a high luminosity up to $\mathcal{L}=10^{30} \mathrm{~cm}^{-2} \mathrm{~s}^{-1}$ and a good resolution $\Delta(\sqrt{s}) \approx 1 \mathrm{MeV}$ for the final-state mass determination. The interaction region is surrounded by a compact detector system which is divided into forward and barrel parts. It is composed of

- a set of 60 scintillation counters arranged around the beam pipe for triggering,

- 2400 straw drift tubes for the recording of charged-particle tracks,

- a 400-wire proportional chamber (MWPC) for supplementary tracking information,

- 5000 silicon-pad counters [15] for the measurement of energy loss of slow charged particles,

- a 5000-cell ring-imaging Cherenkov counter (RICH) [16] providing a velocity measurement of fast charged particles,

- 48 threshold Cherenkov counters for on-line pion rejection,

- a 144-element multilayer scintillator hodoscope [17] for supplementary trigger information,

- 350 electromagnetic shower counters [18] made of lead and scintillating fibres $(\mathrm{Pb} /$ SciFi) for the detection of neutral mesons by their decay photons.

The first-level on-line trigger is based on the multiplicity of charged particles, on the discrimination between particle types $\pi / \mathrm{K} / \mathrm{p}$, on photon multiplicity, and on the specific event topologies of the reactions under study. The $\bar{p}$ p total cross-section of $\sigma_{\text {tot }} \approx 100 \mathrm{mb}$, yields an interaction rate up to $N=\sigma_{\text {tot }} \mathcal{L} \approx 10^{5} \mathrm{~s}^{-1}$ at peak luminosity. As an example, the on-line trigger used for $4 \mathrm{~K}^{ \pm}$final states falling into the geometrical region accepted by the detectors reduces this rate by roughly a factor of 200. Along with the main physics triggers, data on $\bar{p} p$ elastic scattering at large angles are recorded for purposes of normalization and luminosity monitoring. The non-gated total event rate at peak luminosity is typically of the order of $200 \mathrm{~Hz}$ with a nearly $100 \%$ duty cycle of the LEAR machine. 
The JETSET experiment belongs to the category of 'fixed-target' experiments. In many of these cases, the reaction products show a pronounced forward distribution. This effect is sometimes amplified for various reasons. If the produced particles are heavier than those of the initial state, as in $\bar{p} p \rightarrow \phi \phi$ and other 'threshold-type' reactions, the final-state particles, and to some extent also their decay products, are emitted in a more or less narrow forward cone around the beam direction. A 'leading particle' having some resemblance to the incident beam particle tends to follow the direction of the latter, as in the $\bar{p} p \rightarrow \bar{\Lambda} \Lambda$ reaction. On the other hand, $\bar{p} p$ annihilation into multi-pion final states distributes the particles over the whole detector.

\subsection{Implications for the straw-chamber design}

The conceptual design of the straw chamber is driven by specific physics goals and constraints due to the experimental environment. The JETSET detector system is built around a machine beam pipe which forms an oval with full axes of $156 \mathrm{~mm}$ (horizontal) and $76 \mathrm{~mm}$ (vertical). Opening the vacuum tube for installation or removal of the detector is prohibitive owing to the extreme vacuum requirements in a storage ring for low-energy antiprotons.

The straw drift tubes are divided into a forward and a barrel part:

- The forward straw chamber consists of twelve layers of drift tubes perpendicular to the beam axis, six each for horizontal and vertical position sensing. Several blocks of independent modules are arranged in a manner to achieve an approximately circular structure around the beam pipe [19].

- The barrel straw chamber is split into top and bottom halves that are clamped around the beam pipe. Another major complication is caused by the horizontal hydrogen-jet tube of $32 \mathrm{~mm}$ diameter, which runs all the way through the detector and is connected to the beam pipe by bellows of $45 \mathrm{~mm}$ diameter. The corresponding near-midplane space must be filled with special short straw modules ('inserts'), which occupy the regions forward and backward of the jet pipe on both sides of the machine vacuum tube. Because the barrel straw chamber is not positioned symmetrically with respect to the jet tube, the forward and backward short straw modules are different. Thus, the barrel straw-chamber design has three different building blocks, and each of them has to be fabricated twice for the complete detector.

Figure 2 shows an end view of the complete barrel straw detector and fig. 3 a side view of the inserts. The barrel straws are situated in the centre of the JETSET experiment and are inaccessible. Therefore, the reliability of their operation is a major issue.

The drift tubes are oriented parallel to the beam direction. Particles entering the chamber at relatively small angles cause only a short track stub which points through the end plate into the forward straw chamber. Matching the barrel and forward track pieces to a long combined track is only possible if the multiple Coulomb scattering is kept reasonably small. This effect can be reduced by a forward end plate assembly as thin as feasible, which additionally provides track elements as long as possible. The assembly includes short end plugs which keep the wires centred in the tube, the end plate itself which defines the tube position and serves as a gas manifold, and the printed-circuit board which distributes high-voltage and fans out signals to the electronics. Thin end plates imply that the mechanical stability of the chamber must be provided by gluing the tubes together side by side. Thus a rigid barrel straw chamber with a densely packed and almost gapless configuration can be built by using the same diameter and length for all tubes, which also facilitates the calibration of the chamber. 
In order to obtain three-dimensional space information for charged particles passing through the chamber, both transverse and longitudinal coordinates must be read out from the tubes. The required spatial resolutions are $\sigma_{r} \approx 200 \mu \mathrm{m}$ and $\sigma_{z} \approx 8 \mathrm{~mm}$. For the transverse position, this is usually achieved by drift-time measurements, whereas for the determination of the longitudinal coordinate, three different methods are known. We evaluated each of them in view of our requirements and boundary conditions.

- Small-angle stereo. The chamber contains tube layers having a small slant angle $\delta$ with respect to the beam direction, which is typically $2^{\circ}$ or $3^{\circ}$. Drift-time measurements result in a longitudinal accuracy given by $\sigma_{z}=\sigma_{r} / \sin \delta$. Although this method is elegant and conceptually simple, it is not obvious how one can implement it in a barrel-shaped multilayer chamber, where the overall rigidity comes from gluing all the tubes side by side.

- Pick-up electrodes. Inductive azimuthal cathode strips or, alternatively, cathode pads, appear to be very difficult to install and read out in a straw chamber, in particular because they have to be partially transparent for signals. Moreover, this implies a large quantity of electronic channels.

- Charge division. If the anode wire of length $L$ has a sufficiently large resistance, the longitudinal position of a hit can be obtained by using analog readout of both ends of the wire. This method leads to a typical resolution of $\sigma_{z} \approx 0.01 \cdot L$, which is slightly worse than that of small-angle stereo wires. However, the combination of drift-time and charge-division measurements in the same tube directly provides $3 \mathrm{D}$ space points; hence it is very useful for pattern recognition.

We decided in favour of the last method, since a simple way was also found to reduce the electronics effort and the amount of material in the forward direction (see section 3 ).

It is not necessary to operate the straws at high gas pressure; therefore no vessel was foreseen around the chamber. Standard chamber gas at atmospheric pressure is the preferred choice. The gas should have no adverse effects on any material used with the straws, and for safety aspects it should be non-flammable.

\section{Design and construction of the detector}

\subsection{Geometrical and mechanical design}

The JETSET barrel straw chamber has been designed to cover tracks from $\theta \approx 40^{\circ}$ to $150^{\circ}$ and $\phi=0^{\circ}$ to $360^{\circ}$ in polar and azimuthal angles, respectively. Its inner boundary, determined by the size of the beam pipe and the thickness of the scintillator layer surrounding the pipe, is an oval with half axes of $88 \mathrm{~mm}$ (horizontal) and $48 \mathrm{~mm}$ (vertical). The outer diameter of the chamber is $362 \mathrm{~mm}$. Defining $(x, y, z)=(0,0,0)$ as the nominal crossing point of the hydrogen jet and the antiproton beam, the longitudinal position of the chamber in $z$ extends from $188 \mathrm{~mm}$ (forward or downstream) to $-268 \mathrm{~mm}$ (backward or upstream), a total length of $456 \mathrm{~mm}$. All straws have the same outer diameter of $8.0 \mathrm{~mm}$.

Thin-walled aluminium alloy tubes [20] were made by extrusion from carefully milled concentric 'raw' tubes, which had dimensions of $30 \times 24 \times 3 \mathrm{~mm}^{3}$ (outer diameter $\times$ inner diameter $\times$ wall thickness) and a length of $1 \mathrm{~m}$ each. Manufacturing of the straw tubes involved special care in cold drawing operations and subsequent heat treatment. It was our initial aim to obtain straws with a wall thickness not exceeding $30 \mu \mathrm{m}$, either by extrusion down to $60 \mu \mathrm{m}$ and subsequent etching in a bath of caustic soda, or directly from the extrusion process. Neither of the methods turned out to be successful since many of the $30 \mu \mathrm{m}$ tubes showed holes in the metallic surface. Possible reasons are that the 
alloy used had a granularity comparable to the $30 \mu \mathrm{m}$ wall thickness aimed at, or that the extrusion process itself resulted in a non-uniform wall thickness. Clearly, this would cause problems for the gas tightness and also for the strength and symmetry of the electric field. Thus, the nominal wall thickness of the straws was chosen to be $60 \mu \mathrm{m}$, a thickness which could be tolerated from multiple scattering estimates.

Some important parameters of the JETSET barrel straw chamber are summarized in Table ??. They resulted from intensive studies of prototypes as well as subsequent studies with radioactive sources and CERN-PS test beams. One basic concept of the chamber is not to concentrate material in certain regions, but to distribute it over the whole detector.

\subsection{Construction of the straw chamber}

The construction of the barrel straw chamber involved the following main steps.

- Fabrication and selection of tubes. A total of 5000 straws, each $436 \mathrm{~mm}$ long and weighing about $1.7 \mathrm{~g}$, were extruded from 25 'raw' aluminium alloy tubes having a total weight of about $17 \mathrm{~kg}$. The tubes were cleaned in an ultrasonic bath and then visually inspected for mechanical flaws such as scratches or dents. 'Good' tubes were finally selected by measuring the wall thickness all around the circumference at three positions along the tube. For the detector construction we retained only those tubes having a wall thickness $d=(60 \pm 5) \mu \mathrm{m}$.

- Gluing of sub-blocks. About 30 to 40 straws at a time were straightened by inserting into each of them a high-precision steel rod. These tubes were then placed in a machine which moved a nozzle along the tube and laid strings of cyanoacrelate glue [21] droplets on the outer surface. A straw that was going to have six neighbouring tubes received three glue strings separated by $120^{\circ}$ in azimuth. Precisely machined plates locked the steel rods - and also the tubes - in accurate positions relative to each other until the glue had dried. For the two barrel halves, in this way, we fabricated 17 sub-blocks each, plus the special near-midplane modules.

- Length adjustment. After removal of temporary end plates and steel rods, a plastic ring of a few millimetres length was covered with glue on its outer surface and inserted into each end of a tube. This ring constitutes one of two end plug pieces [22]. Its main purpose here is to be positioned in such a way that all tubes obtain exactly the same mechanical length and are therefore flush against the end plates. A photograph of a sub-block partially equipped with its end plugs is shown in fig. 4.

- Assembly of barrel-halves. The final end plates of the barrel-halves were mounted on a precision support structure, which ensured that they were parallel and had zero relative rotation angle in all planes. These end plates were fabricated from $7 \mathrm{~mm}$ thick aluminium alloy material. A total of 597 high-precision two-step holes with final dimensions $\varnothing 8.4 \times 5 \mathrm{~mm}^{2}$ and $\varnothing 6.0 \times 2 \mathrm{~mm}^{2}$ were drilled by a numerical boring machine. One face of the end plates - the inner one, where they touch the straw tubes - is flat. The other face - the outer one, which would serve as a gas manifold has between any three $\varnothing 8.4 \mathrm{~mm}$ holes a triangular post sticking out of the base plate, as shown in fig. 5. The effective remaining thickness of the base plate is thus only $2 \mathrm{~mm}$. All 17 sub-blocks belonging to one half of the barrel were suitably equipped with strings of glue and accurately locked in position between the end plates until the glue had hardened.

- Insertion of wire feedthroughs. The tube end plugs have several functions. They determine the exact wire position in the straw, provide electrical insulation between 
the cathode wall and the anode wire, and also allow the gas to enter and exit the tube easily. The plastic end plug [22] consists of an outer ring and an inner wire feedthrough. The latter has the important feature of a V-shaped groove, which forces the anode wire to be precisely centred in the straw tube. The feedthroughs are inserted into the end plate and the outer ring (already mounted on the straws) such that the $V$-groove points towards the horizontal midplane of the chamber, i.e. 'downwards' during the construction phase. A cross-sectional view of the end plug and surrounding items are shown in fig. 6. A photograph of a partially equipped end plate is shown in fig. 7.

- Mounting of printed-circuit boards. The printed-circuit boards define the electrical end of a single anode wire. For each wire there is a conical metallized hole, its edges being rounded-off so as to avoid too small a bending radius of the anode wire. The design of the printed-circuit boards is very different for the front ('downstream') and rear ('upstream') plates. Each of the two rear half-plates carries 597 'male' pins soldered onto it in order to facilitate the electrical connection to each anode wire. On each front half-plate are soldered about 295 small resistors in 'surface-mount device' (SMD) technology. We have chosen $R=47 \Omega$, which is equivalent to about $5 \mathrm{~cm}$ of anode wire. Each of these resistors connects two straw anode wires. To keep the full 3D space information of all hits, a particle emitted from the target region must pass through only one straw of a pair. Therefore the connections between two wires are made in a way that the plane defined by these two wires is tangential to a cylinder which is symmetric around the beam axis. In the off-line analysis, the hit straw is unambiguously identified by the larger of the two measured pulse heights.

The printed-circuit boards are screwed against the outer face of the aluminium alloy end plates, and both pieces together form a gas-tight sandwich-type manifold for low-impedance gas distribution and gas flow in all straws.

A special multi-strip Kapton cable connects the forward and backward parts of the near-midplane modules, which are separated by the gas-jet pipe. SMD resistors of $R=47 \Omega$ are mounted on the Kapton cable, providing a resistance corresponding to an anode wire of the gap's length.

- Wire stringing. Wire for gaseous chambers must have excellent mechanical and chemical properties. The localization of a particle along the wire through resistive charge division requires a large wire resistivity. We have chosen stainless-steel wire [23], since good results have already been obtained in similar applications [24]. The nominal wire diameter is $35 \mu \mathrm{m}$, which was confirmed by optical measurements with an uncertainty of about $1 \mu \mathrm{m}$. Its resistivity is about $1 \mathrm{k} \Omega / \mathrm{m}$. Pieces of $1 \mathrm{~m}$ length were spooled off a reel, cut, and cleaned. The wire was introduced into the metallized conical hole of the printed-circuit board and into the feedthrough of the straw. It was subsequently pushed and pulled through the tube with the help of magnetic needles until it reached the other side of the chamber. A weight was attached to each end of the wire. This resulted in a tension of $T \approx 0.9 \mathrm{~N}$, which during tests had been found to be an appropriate value for our purposes. A metallized plastic rivet [22] was pressed into the conical hole of the printed-circuit board at each end of the wire. This fixed the wire and also guaranteed good electrical contact between the wire and the printed-circuit board. We estimate that the end plugs centre the wires with an accuracy corresponding to the wire diameter. Since the straws are relatively short, the wires only need to be supported at the tube ends. In each of the two barrel-halves eight tubes are not equipped with wires, but used for gas-return flow to the rear end 
plate.

- Mechanical protection. For detector testing and installation, the aluminium alloy tubes must be protected against mechanical damage. The outer and inner surfaces of the barrel-halves, as well as the sides of all near-midplane modules, are therefore covered with a thin layer of Balsa-wood/fibre-glass laminate forming a very rigid 'skin'. A photograph of a completed detector half is shown in fig. 8.

\subsection{Mechanical and electrical properties of the chamber}

After the end of the mechanical construction, the straw chamber had to undergo some basic tests. These included checks of gas tightness, anode-wire characteristics, and high-voltage behaviour. All anode wires showed very large insulation resistances with respect to the cathodes $\left(>2 \times 10^{10} \Omega\right)$. Measurements along the barrel wires yielded a single-wire resistance of $R_{\text {wire }}=(466.4 \pm 3.0) \Omega$, a jumper resistance of $R_{\text {jumper }}=(46.7 \pm$ 1.0) $\Omega$, and a total resistance of a jumpered wire pair $R_{\text {pair }}=(978.5 \pm 4.2) \Omega$. In the case of the wire 'pairs' of the near-midplane modules, the resistances of the four short anode wires, the Kapton connections, and the three jumper resistors summed up to a total value of $R_{\text {pair }}^{\prime}=(954.9 \pm 14.2) \Omega$.

The wire tensions of all straws were measured by the method of acoustic resonance in a magnetic field. A sinusoidal current was driven through the wire in the presence of a magnetic field perpendicular to it. The force on the wire creates a mechanical oscillation, which in turn induces an electromagnetic field along the wire. The driving current and the induced voltage are monitored with an oscilloscope and are used to detect the resonance condition when varying the oscillator frequency. The $n$th harmonic of the standing waves has a frequency

$$
\nu_{n}=\frac{n}{2 L} \sqrt{\frac{T}{\rho}},
$$

where $L$ denotes the wire length, $T$ the wire tension, and $\rho$ the wire mass per unit length. The measurements resulted in $T=(0.87 \pm 0.06) \mathrm{N}$.

The different materials contribute to the thickness and thus to the radiation length as follows:

- Aluminium. The average thickness $x_{\text {tube }}$ of a straw per layer is given by the crosssection of a straw divided by the straws' spacing, i.e. $x_{t u b e}=187 \mu \mathrm{m}$.

- Stainless-steel wires. The wire radius of $17.5 \mu \mathrm{m}$ results in $x_{\text {wire }}=0.12 \mu \mathrm{m}$ per layer.

- Glue. Assuming that each straw glue joint has an average cross-section of $6000 \mu \mathrm{m}^{2}$, three joints of glue per straw cause a thickness per layer of $x_{\text {glue }}=2.25 \mu \mathrm{m}$.

- Gas. For the chosen 50/50 mixture of $\mathrm{Ar}$ and $\mathrm{CO}_{2}$, both gases contribute equally to the thickness inside the drift tubes. Each counting gas adds $x_{\text {gas }}=3048 \mu \mathrm{m}$ per layer. Air is assumed to fill the remaining area outside, resulting in $x_{\text {air }}=968 \mu \mathrm{m}$ per layer.

In Table ?? the total thicknesses and the respective fractions of the corresponding radiation lengths are given. For the calculation it is assumed that the tracks have passed through 15 layers of straws on an average. The thickness and the radiation length are given for orthogonal tracks. Thus, a track with $\theta=40^{\circ}$, which is the longest possible passing through the barrel, traverses $5 \%$ radiation lengths. 
4 Amplifier and discriminator electronics

\subsection{Principal concepts}

All connections of the barrel straw chamber with external equipment, such as gas and high-voltage supplies, are made from the backward (upstream) end plate. In that region, however, the JETSET detector system does not provide sufficient free space for mounting the chamber front-end electronics. Therefore, cables must transmit the signals over some distance.

The anode wire of each straw connects to the central conductor of a $3.5 \mathrm{~m}$ long miniature coaxial cable [25]. This cable has an outer diameter of $1.22 \mathrm{~mm}$ and a characteristic impedance of $80 \Omega$. Its resistance and capacitance have been measured to be $5 \Omega / \mathrm{m}$ and $60 \mathrm{pF} / \mathrm{m}$, respectively. The cable transmits wire signals to the preamplifier, and it also supplies the straw anodes with positive high voltage. We have observed no indication of discharge or a sizeable leakage current during long-term tests, where a $100 \mathrm{~m}$ cable was subjected to $4 \mathrm{kV}$, almost twice the normal operating voltage. Groups of sixteen cables are formed and their outer conductors are clamped together and connected to a central grounding point at the end plate. A total of about 1400 cables, carefully tied together in one single bundle, runs to the rack-mounted preamplifier electronics located $3 \mathrm{~m}$ away from the detector. Here the cables are connected in 16-channel groups to the preamplifier and high-voltage circuitry via small adapter cards. We have found no sizeable high or low-frequency noise due to this overall arrangement, which may be just a consequence of favourably chosen electrical and geometrical configurations. The small noise level is remarkable, particularly since the experiment is installed in a compact storage ring with accelerating cavities and equipment for phase-space cooling.

Some basic features of the front-end electronics are summarized in Table ??. In fig. 9, the scheme of front-end signal transmission is shown for one pair of straws, from the straw anode wires through the preamplifiers and postamplifier/discriminator units to the charge- and time-digitizing readout electronics. To avoid long coaxial cables, the preamplifiers are situated close by the detector in the LEAR machine area, whereas all other electronics is located in the main counting room of the experiment. The quasicomplementary preamplifier output drives a $20 \mathrm{~m}$ long twisted-pair cable, where 32 signal pairs $(+$ and -$)$ are surrounded by a common grounded shield. The signals are then fed into a differential postamplifier, which is followed by two circuits to perform chargedivision and drift-time measurements. A differential current amplifier drives a 32-channel shielded twisted-pair cable, which is $60 \mathrm{~m}$ long and provides a signal delay of about $300 \mathrm{~ns}$ for analog-to-digital converters (ADCs). A fast discriminator with complementary ECL outputs drives a $5 \mathrm{~m}$ long 16-channel twisted-pair flat cable which connects to time-todigital converters (TDCs) and is also used to generate higher-level trigger decisions. All the ADC and TDC readout modules are of Fastbus standard, and they are connected by high-speed interfaces to a data-acquisition system based on the VMEbus standard [26].

\subsection{Preamplifier}

The 16-channel preamplifier card (PA, type FPF-228B) is designed [27] around the Fujitsu MB43468 monolithic charge-sensitive amplifier [28]. This chip has four channels housed in a 14-pin SMD package and requires only a few external components. Each preamplifier board contains four of these chips. The input is externally protected by a $10 \mathrm{nF}$ high-voltage blocking capacitor, a series resistor, and a pair of antiparallel shunt diodes. A shunt resistor of $120 \Omega$ matches the impedance at the input of the preamplifier card with the $80 \Omega$ impedance of the miniature coaxial cable. The Fujitsu chip converts 
a negative-input charge pulse into a positive-output voltage pulse. A pair of resistors transforms the latter into quasi-complementary signals which run through twisted-pair cables to the counting room. The overall preamplifier gain, for a charge injected into the $3.5 \mathrm{~m}$ long miniature coaxial cable and an output loaded with $2 \times 56 \Omega$, is measured to be $-215 \mathrm{mV} / \mathrm{pC}$. Typical output signal rise and fall times are $t_{\text {rise }}=6 \mathrm{~ns}$ and $t_{\text {fall }}=30 \mathrm{~ns}$, respectively, which turns out to be well adapted to the detector characteristics. The crosstalk measured for the preamplifier board is less than $2 \%$, and the peak-to-peak noise level is about $10 \mathrm{mV}$.

High-voltage is applied to a SHV connector mounted on the preamplifier card and is fanned out with $10 \mathrm{M} \Omega$ series resistors to the individual straw anodes. A $100 \mathrm{k} \Omega$ resistor separates the 'high-voltage ground' from the local signal ground to avoid ground-loop problems. All channels of a board can be checked qualitatively with a test signal that is fanned out to the 16 preamplifier inputs. This test circuit converts a $1 \mathrm{~V}$ step into charges of about $1 \mathrm{pC}$.

The electromechanical standard of the PA boards is the Europa format with a height of $265 \mathrm{~mm}$ ( $6 \mathrm{U}$ ), a width of $20 \mathrm{~mm}$ (4 TE), and a card depth of $160 \mathrm{~mm}$. Twenty preamplifier cards are located in a crate and are supplied with $+8 \mathrm{~V}$ from the back-plane connector under the control of a PA-power card (FPF-230B). We have built eight crates loaded with a total of 160 preamplifier boards, thus comprising 2560 electronic channels for the barrel and forward straw chambers plus some spares. The power consumed by this whole system is only $8 \mathrm{~V} \times 8 \mathrm{~A}$ and can thus be supplied by a relatively small $10 \mathrm{~A}$ external power pack.

\subsection{Postamplifier and discriminator}

The 16-channel postamplifier/discriminator card (PAD, type FPF-227B) [27] fulfils several functions. After transmission through $20 \mathrm{~m}$ of cable, the complementary preamplified signals are received and further amplified by National Semiconductor LM733 CN chips, a differential voltage amplifier. The gain is determined by the ratio of two external resistors, in our case $22 \mathrm{k} \Omega / 1.5 \mathrm{k} \Omega \approx 14.6$. The output of this amplifier stage is split into two branches referred to as analog and digital.

In the analog branch, single-stage current amplifiers consisting of a 2N3904-2N3906 transistor pair drive the complementary signals through $60 \mathrm{~m}$ twisted-pair cables to ADC inputs, which are symmetrically terminated with $2 \times 56 \Omega$. The overall gain of the whole analog chain, measured from the chamber-connected miniature coaxial cable all the way through the various amplifier stages to the ADC input and including all losses in cables, is $\pm 200 \mathrm{mV} / \mathrm{pC}$. The system has good linearity up to about $3 \mathrm{pC}$ input charge. Typical signal characteristics at this point, where timing is not important, are $t_{\text {rise }}=16 \mathrm{~ns}$ and $t_{\text {fall }}=40 \mathrm{~ns}$. The analog cross-talk measured for the postamplifier/discriminator board is below $5 \%$, and the peak-to-peak noise level is less than $\pm 50 \mathrm{mV}$.

The central piece of the digital branch is a LeCroy MVL407 high-speed discriminator [29]. This is a four-channel voltage-comparator chip housed in a 20-pin DIP package, featuring transition times around $1 \mathrm{~ns}$ and toggle frequencies up to $400 \mathrm{MHz}$. The PAD board carries four such chips with differential inputs and complementary ECL outputs. The negative input pulse has a zero base line, whereas the base line of the positive input pulse can be adjusted externally between 0 and $-1 \mathrm{~V}$. If the positive and negative input signals overlap, the discriminator switches its state to give complementary ECL output signals. Their width is determined by the time-over-threshold condition of the input pulses. In reality, a $20 \mathrm{mV}$ input overlap is needed to produce a minimum output 
width of about 9 ns.

The charge $Q_{\text {in }}$ required at the miniature coaxial cable to switch the state of the discriminator is, for a threshold setting $U_{t h r}$, approximately given by

$$
Q_{\text {in }} \approx 0.45 \frac{p C}{V} \cdot U_{t h r}-0.02 p C \text {. }
$$

A typical running condition is $U_{t h r}=-0.2 \mathrm{~V}$, which corresponds to a lower limit of $Q_{\text {in }} \approx-0.11 \mathrm{pC}$ delivered by an anode wire.

The PAD card provides two kinds of digital outputs. Each individual channel has a 'direct' output which can be connected to a TDC or, alternatively, to higher-level trigger logic. In addition, any two neighbouring channels are cabled so that they belong to a pair of jumpered straws, being just ORed after the discriminator stage using MC10103 and MC10104 circuits. This common 'multiplexed' output is connected to the TDC, the idea being that in the case of a hit straw only the first signal recorded from a pair is relevant for the actual drift-time information.

The electromechanical standard of the PAD boards is the Europa format with a card depth of $220 \mathrm{~mm}$. Twenty PAD cards are located in a crate and supplied with $\pm 5 \mathrm{~V}$ from the back-plane connector under the control of a PAD-power card (FPF-232B) and appropriate relays. We have built eight crates loaded with a total of 160 PAD boards, thus comprising 2560 electronic channels for the barrel and forward straw chambers including some spares. The power consumption of the whole system is $+5 \mathrm{~V} \times 80 \mathrm{~A}$ and $-5 \mathrm{~V} \times 160 \mathrm{~A}$, thus totalling $1200 \mathrm{~W}$. For the supply we use four external $100 \mathrm{~A}$ power packs.

The voltages determining the discriminator thresholds are provided by a remote threshold-control and display unit (type FPF-234B). A common threshold voltage is distributed from the PAD-power card via the crate back-plane connectors to the PAD boards. Alternatively, the thresholds can be set locally on each PAD card.

For the $20 \mathrm{~m}$ long connection from the preamplifier outputs to the postamplifier/ discriminator boards, and for the $60 \mathrm{~m}$ long connection from the postamplifier analog outputs to the ADCs, 32-channel shielded twisted-pair round cables are used. These are split at each end into two 16-channel branches and equipped with standard 34-pin flatcable connectors. The discriminator ECL outputs are connected to the TDCs by $17-$ channel twisted-pair flat cables of $5 \mathrm{~m}$ length. The whole installation comprises about $6.5 \mathrm{~km}$ of multi-channel signal cables.

\section{Operation, calibration, and performance}

\subsection{Operational conditions}

Owing to the basic design and the experimental concept, the operational conditions of the chamber turn out to be quite moderate. Consider a straw tube of radius $r=4 \mathrm{~mm}$, its wire running parallel to the beam direction at a radial distance $R$ from the beam. For an infinitely long wire, the hit rate of a reaction with cross-section $\sigma$ at a luminosity $\mathcal{L}$ is given by

$$
\frac{d n}{d t}=\frac{r n_{c} \sigma \mathcal{L}}{\pi R},
$$

with $n_{c}$ denoting the charged-particle multiplicity per interaction. For a typical drift velocity $v_{D}$ of the electrons, the maximum drift time is $r / v_{D} \approx 250 \mathrm{~ns}$. The single-wire occupancy due to electron drift thus becomes

$$
O=\frac{r^{2} n_{c} \sigma \mathcal{L}}{\pi R v_{D}} .
$$


Each particle traversing the tube at an angle $\theta$ produces an amount of ionization up to $2 r \alpha_{t} / \sin \theta$, where $\alpha_{t}$ is the total ionization per unit length in the chamber gas. For the barrel straws, the angle $\theta$ is always $\theta \geq 15^{\circ}$, therefore the maximum current drawn by a wire operated with gas amplification $G$ is

$$
I=\frac{8 r^{2} n_{c} \sigma \mathcal{L} e \alpha_{t} G}{\pi R}
$$

With $R \geq 5 \mathrm{~cm}$, and assuming $n_{c}=4, \alpha_{t}=100 \mathrm{~cm}^{-1}$ and $G=10^{5}$, we obtain at peak luminosity the single-wire 'worst-case' upper limits for the hit rate $d n / d t \leq 10^{4} \mathrm{~s}^{-1}$, the occupancy $O \leq 0.1 \%$, and the current $I \leq 50 \mathrm{nA}$. None of these figures is critical; therefore questions of the chamber lifetime appear not to be relevant. It should be noted, however, that the hit distribution along the wire is strongly peaked in the forward direction.

Since the charge-division method is used in addition to the drift-time readout, operating parameters such as the gas mixture, the gas pressure, and the high voltage have to be a compromise to ensure good results for both techniques and cannot be optimized for any one of them. The choice of the operating parameters was based on numerous tests performed in the laboratory with X-ray sources as well as at the CERN-PS test beam [30]. The behaviour of several prototypes has been studied, from single straws to complex multi-tube arrangements corresponding to a $30^{\circ}$ sector of the whole barrel straw chamber.

Gas. Most straw chambers use mixtures of noble gases such as argon and organic gases such as ethane or dimethylether (DME). Our tests showed that good results could be achieved with an $\mathrm{Ar} / \mathrm{CO}_{2}$ mixture, which is also safer in the sense that it does not contain flammable ingredients. It was found that for our purposes there is no need to work with pressurized gas. We operate the straws at room temperature and atmospheric pressure, which allows safe and reliable working conditions. The gas flow and mixture, at present a 50/50 mixture, is determined by electronic mass-flow regulators of the type 5850 TR together with a 5878 control unit [31]. To provide a sufficient gas flow through the straws, even in case of some leakage being present, bubblers were installed in the return line for visual control. The gas flow under normal working conditions is about $150 \mathrm{~cm}^{3} / \mathrm{min}$, replacing the active gas volume every three to four hours. Further studies were carried out to explore the behaviour of the straws with different gas mixtures.

High voltage. Using $\mathrm{CO}_{2}$ as the chamber gas, the straw-anode high-voltage was ramped up to $+3.2 \mathrm{kV}$ without problems. Being safe from this point of view, rate measurements have been performed with $5.9 \mathrm{keV}$ X-rays from a ${ }^{55}$ Fe source to determine the adequate operating voltage. The straws are at present operated at $2150 \mathrm{~V}$. This value corresponds to the lower part of the plateau characterizing the proportional mode of the $50 / 50$ mixture of $\mathrm{Ar} / \mathrm{CO}_{2}$, which extends up to about $2300 \mathrm{~V}$. The chosen voltage ensures stable operation with a reasonable gas amplification of some $10^{5}$ without the risk of sparks or the production of streamers.

Efficiency. A charged particle traversing a gas volume produces primary ionization clusters, whose number is governed by a Poisson distribution. The detection inefficiency is therefore given as

$$
1-\epsilon=\exp \left(-\frac{\alpha_{p} l}{p / b a r}\right),
$$

where $l$ is the track length, $p$ is the gas pressure, and $\alpha_{p}$ denotes the density of primary ionization pairs at standard temperature and pressure (STP). For our 50/50 mixture of 
Ar and $\mathrm{CO}_{2}$ we expect $\alpha_{p}=31.8 \mathrm{~cm}^{-1}$, which would lead to $1-\epsilon=10^{-9}$ for the average path length of $6.2 \mathrm{~mm}$ and $1-\epsilon=10^{-12}$ for the maximum path length of $8 \mathrm{~mm}$ through a straw. Even tracks passing $10 \mu \mathrm{m}$ from the wall, corresponding to a path length of $0.6 \mathrm{~mm}$ would have an inefficiency of less than $15 \%$.

\subsection{Readout}

The readout principle, together with the electronics used, has already been described in section 4 and is shown in fig. 9. Whereas the drift-time measurement is done via a single TDC per wire pair, for the charge-division read-out both ends of the resistive wire pairs are connected to individual ADCs.

The electronic connection scheme of a wire pair is shown in fig. 10. As mentioned before, the two straws forming a pair are connected via a jumper resistor to form a single charge-division system. The ends of the wire pairs are each connected via $3.5 \mathrm{~m}$ of miniature coaxial cable [25] to the input of the preamplifiers.

Drift-time information. The timing signal for the drift-time information of the straws is generated by the PADs. The usual threshold under running conditions is $-200 \mathrm{mV}$ which corresponds to a charge of about $7 \times 10^{5}$ electrons collected at the end of an anode wire. These signals are used to start the Fastbus TDCs. The timing reference is obtained from the trigger scintillators placed around the beam pipe, provided certain trigger conditions are matched. This signal, delayed by $600 \mathrm{~ns}$, then acts as a common stop for the Fastbus TDCs.

Measurements done with a staggered four-tube array showed resolutions which improved from about $250 \mu \mathrm{m}$ for very short drift radii (i.e. some hundreds of microns) to $\sim 50 \mu \mathrm{m}$ for rather long drift radii. The latter value corresponds well to the calculated limit by diffusion. We can therefore estimate an average resolution of $\sim 180 \mu \mathrm{m}$. It should be noted that this transverse resolution is ultimately limited by fluctuations of the ionization density, the electron diffusion, and finally the accuracy of the drift-time measurement. The resolution is expected to decrease with decreasing incident angle with respect to the straw axis. This is due to longer path lengths in the counting gas.

Charge-division readout. Figure 10 shows the principle and the notation used. The position of the current source can be calculated using the currents $i_{1}, i_{2}$ received at the preamplifiers PA1, PA2. It is convenient to use a signed coordinate $\xi$, running along the resistive length and having its origin at the centre, i.e. the 'midpoint' of the jumper resistor. The coordinate of the current source is then given by

$$
\xi=\frac{R_{2}+R_{1}}{2 \rho} \frac{i_{2}-i_{1}}{i_{2}+i_{1}}+\frac{R_{2}-R_{1}}{2 \rho},
$$

where $R_{1,2}=\left(R_{\text {cable } 1,2}+R_{\text {wire } 1,2}+\frac{1}{2} R_{\text {jumper }}\right)$ and $\rho$ is the specific resistance of the anode wire. The second term reflects possible asymmetries. The relationship between $\xi$ and the physical coordinate $z$ as used in the laboratory system is given by $z=z_{\text {end }} \pm \xi$, the sign depending on which wire was hit and $z_{\text {end }}$ being the physical coordinate of the forward end of the barrel straws. Equation (7) also holds if the currents are replaced by the respective charges $q_{1}, q_{2}$ collected during a certain period of time, as used in our experiment.

Taking differences in the amplifier gains $g_{1}, g_{2}$ into account, the difference-to-sum ratio $\zeta$ of the observed charges $Q_{1}, Q_{2}$ can be related to the ideal case by

$$
\zeta=\frac{Q_{2}-Q_{1}}{Q}=\frac{g_{2} q_{2}-g_{1} q_{1}}{g_{2} q_{2}+g 1 q_{1}}=\frac{q_{2}-\alpha q_{1}}{q_{2}+\alpha q_{1}},
$$


thus having introduced $Q=Q_{1}+Q_{2}$ and the asymmetry parameter $\alpha=g_{1} / g_{2}$.

We can expand $\xi$ to be

$$
\xi=\sum_{n=0}^{\infty}\left(a_{n} \zeta^{n}+b_{n} Q^{n}\right),
$$

where the coefficients can be expressed as a power series of the asymmetry. Assuming $\alpha=1 \pm 0.1$ and aiming at an uncertainty for $z$ of about $1 \%$, the expansion in $\zeta$ can be cut after the second-order term. For the correction term depending on $Q$, only the linear term was found to be necessary. Rearranging the above equation, $\xi$ can finally be expressed as

$$
\xi=c_{1}\left(\zeta-\zeta_{0}\right)+c_{2}\left(\zeta-\zeta_{0}\right)^{2}+\Delta \xi(Q),
$$

with $\Delta \xi(Q)=b_{0}+b_{1} Q$. The values of the parameters $\zeta_{0}, c_{1}, c_{2}, b_{0}$, and $b_{1}$ have now to be determined for each wire pair.

\subsection{Calibration}

Drift time. The calibration of drift-time as a function of radial distance is done to first order by the method of homogeneous irradiation, using the equation

$$
r(t)=r_{\text {wire }}+\int_{0}^{t} n(t) d t \frac{r_{\text {straw }}-r_{\text {wire }}}{\int_{0}^{T} n\left(t^{\prime}\right) d t^{\prime}}
$$

where $r_{\text {straw }}$ is the inner radius of a straw tube (i.e. $3940 \mu \mathrm{m}$ ), $r_{\text {wire }}$ the radius of an anode wire (i.e. $17.5 \mu \mathrm{m}$ ), and $T$ the maximum drift time. The latter is of the order of $250 \mathrm{~ns}$.

This rather coarse method was applied to a sample of elastic $\bar{p} p$ scattering events. These were selected by demanding a well-determined forward track and a barrel track which fell within the scattering plane. After a linear fit, requiring at least ten hits per track, the residuals $d=r_{\text {measured }}-r_{f i t}$ were recorded. The coordinate $r_{\text {measured }}$ is given by eq. (11) and $r_{f i t}$ is the corresponding radius predicted by the fit. A Gaussian fit to the resulting residual distribution results in a $\sigma_{r}$ of about 500 to $600 \mu \mathrm{m}$, which is significantly larger than the estimated average resolution of about $180 \mu \mathrm{m}$.

Examining the different contributions to the large uncertainty, the effect of time jitter in the start signals of the individual straws and the stop signals of the individual scintillators for the drift-time measurement was found to have the biggest influence. Thus it was essential to look at all individual drift-time spectra corresponding to single straws and to single-trigger scintillators, in order to correct for the time offsets, which showed differences of up to $10 \mathrm{~ns}$.

The relation obtained for $r(t)$ is common for all straws. It is expressed by fitting a 7th-degree polynomial. A recalibration is then performed using the iteration equation

$$
r_{f i t}^{(n+1)}\left(t_{i}\right)=r_{f i t}^{(n)}\left(t_{i}\right)-\left\langle d^{(n)}\left(t_{i}\right)\right\rangle .
$$

Here $r_{f i t}^{(n)}\left(t_{i}\right)$ is the $n$th iteration of the drift distance as a function of the time bin $t_{i}$ and $\left\langle d^{(n)}\left(t_{i}\right)\right\rangle$ is the mean value of the respective residuals. The calculation is done for all relevant time bins and afterwards a new smoothing procedure yields the corrected relation for $r(t)$. The iteration is terminated when the $\left\langle d^{(n)}\left(t_{i}\right)\right\rangle$ values are sufficiently small, which is normally reached after about five steps. Figure 11 shows the final calibration curve, together with the starting values from homogeneous irradiation.

Using the final calibration we can now extract, from a Gaussian fit to the residual distribution, an average value of $245 \mu \mathrm{m}$ for $\sigma_{r}$. Looking at different drift radius regions, 
this corresponds to values from about $480 \mu \mathrm{m}$ for very short radii to about $140 \mu \mathrm{m}$ for larger ones, as shown in fig. 12 .

Charge division. For each wire pair the values of the parameters $\zeta_{0}, c_{1}$, and $c_{2}$ of eq. (10) have to be determined. As a consistency check at the end of the calibration procedure, $z_{\text {end }}$ was treated as an additional free parameter. In a first-order ansatz, the correction term $\Delta \xi(Q)$ was considered negligible. Four calibration points for each wire pair are thus needed and the obvious choice would be the forward and backward physical limits of the active region of each individual wire. These should be visible in the $\zeta$ distribution of all hits on a wire pair.

Since the trigger counters are installed in the forward hemisphere, only the two forward limits can be easily identified. To overcome the lack of a sufficient number of backward-going tracks, an additional trigger was implemented. A ring of scintillator was fixed symmetrically around the $z$ axis. It triggered on tracks from the target at a laboratory angle of about $140^{\circ}$. The special data sample thus produced yielded the $\zeta$ distributions needed for the determination of the calibration parameters.

The error in the $z$ position is expected to be proportional to $\sigma_{Q} / Q$, with three main contributions to $\sigma_{Q}[32]$ :

- the position-independent Johnson noise of the resistive anode wire;

- the electronic noise from the amplifiers;

- the spatial distribution of avalanches due to track inclination.

Assuming $\sigma_{Q}=\sigma_{Q_{1}}=\sigma_{Q_{2}}$ and the correlation between the noise on $Q_{1}$ and $Q_{2}$ to be negligible, we derive from eq. (10)

$$
\sigma_{z} \approx \sqrt{2} c_{1} \sigma_{Q} / Q
$$

The value of $\sigma_{Q}$ can be estimated from the ADC pedestal widths, which are of the order of five channels. Thus a $\sigma_{z}$ of a little below $0.8 \mathrm{~cm}$ can be expected for minimum ionizing particles.

A test of the actual $z$ resolution was performed using a similar sample of clean elastic-scattering events as for the drift-time calibration. A linear least-squares fit was applied to the data, requiring at least ten hits per track. Then the residuals $d=z_{\text {measured }}-z_{\text {fit }}$ were recorded, with $z_{\text {measured }}$ being the coordinate given by the charge division and $z_{f i t}$ the corresponding value from the linear fit. A Gaussian fit to the resulting residual distribution yields a $\sigma_{z}$ which is a little larger then the expected value assuming only noise contributions to the uncertainty. A look at the $Q$ dependence of $\sigma_{z}$ finally revealed a systematic error of about $0.4 \mathrm{~cm}$, which adds up to a total $\sigma_{z}$ of $0.83 \mathrm{~cm}$, as can be seen in fig. 13 as a function of the $z$ coordinate. This corresponds to less than $1 \%$ of the total wire length, a value which seems to be the best attainable resolution obtained by other experiments.

\subsection{Test possibilities}

The preamplifiers FPF-228B, already described in section 4.2, offer the possibility of connecting a test signal to all 16 channels via a single front-panel connector. This signal is distributed by a strip line with $1 \mathrm{pF}$ capacitance to the inputs of the integrated amplifiers MB43468.

To fully exploit the features of these test inputs, a dedicated system consisting of a pulser (type FPF-249B) [33] together with two 80-fold analog fan-outs (type FPF247B) [34] has been installed. The test pulser produces signals with short rise times of 
the order of some nanoseconds and rather long fall times, e.g. $100 \mu$ s. Their amplitude is continuously selectable in the range between $10 \mathrm{mV}$ and $2.50 \mathrm{~V}$ via a potentiometer; the frequency can be chosen in steps between $1 \mathrm{~Hz}$ and $10 \mathrm{kHz}$. The output can be used to simulate charge pulses to feed into a charge sensitive preamplifier. In our case this is done via 80 -fold fanouts, which provide the distribution to the test inputs of the 16-channel preamplifiers.

Since the test pulser additionally supplies a gate input as well as a synchronization output, the whole system can be used with the data acquisition running. For a suitably generated trigger, it is possible to check the functioning of all channels simultaneously, both the TDC and ADC branches. This is very important to allow a reliable operation during the running of the experiment.

\subsection{Performance in experiment}

A scheme of the trigger logic for the straws' readout is shown in fig. 14. Two main branches can be associated with the first- and second-level trigger.

The first-level trigger can be selected to be either the general experimental trigger (physics trigger) from the main logic, a random trigger, generated via a noise pulser of type FPF-102B [35], or a test trigger, synchronized with the signals of the test pulser described in the previous subsection. At this stage, the timing is critical, since the firstlevel trigger signals are available only about $250 \mathrm{~ns}$ after arrival of the detector signals at the electronic inputs. The gate signals for the Fastbus ADCs are generated using the earliest trigger signal. From the first-level trigger also the common stop for the Fastbus TDCs is derived, allowing a time window of $600 \mathrm{~ns}$. Thus the drift times which are of the order of 250 ns easily fit into this range.

Each first-level trigger is followed either by a second-level trigger or by a fast-clear signal in the case where the event is rejected.

- If a second-level trigger signal arrives, it will be about $45 \mu$ s after the corresponding first-level signal. The main action caused by the second-level trigger is an interrupt sent, via a CAMAC input register, to the data-acquisition system. This will in turn read out the various Fastbus and CAMAC modules. Additionally, a hardware clear for these modules is generated as soon as the readout procedure has ended.

- If a fast clear arrives, it immediately executes the hardware clear.

When the system is responding to the random or test trigger, these are then also routed to the second-level logic. Thus, all the electronics can easily be used for test purposes, without the need to change timing, etc. The random trigger, without physics signals being present, is used to determine the ADC pedestals. This is necessary not only to estimate the noise, but also to implement pedestal subtraction and zero suppression.

A complete overview of the data-acquisition system as well as a detailed description of its components and their operation is given in ref. [26].

\subsection{Application in second-level trigger}

The straws signals as given by the PADs are available rather early compared to the physics trigger signal. Together with the fact that all detector information is read out from the respective Fastbus modules some time later, this opens up the possibility of deriving a fast trigger decision based upon information from the straws.

Since the straws are the essential tracking device in the JETSET experiment, events having too few hits - and thus without reliably reconstructible tracks - offer themselves as the first choice for being rejected on-line. This is more important in the case of the 
forward straws, which are separated into four planes, two with vertically and two with horizontally oriented straws. For satisfactory track reconstruction a sufficient number of hits from both projections is needed there.

As a first step towards a second-level trigger, the hit multiplicities of the four forward planes were used to generate a Fast Clear. Off-line studies of these multiplicities showed a potential of reducing the accepted trigger rate by about $40 \%$, whereas only a negligible percentage of 'good' reconstructible events would be lost. This can be achieved by simply rejecting those events which do not have a minimum hit multiplicity in all of the four planes of the forward straws.

The channels corresponding to the four planes are grouped together all along the amplifier and discriminator electronics. Thus their hit multiplicity can be determined using the ECL outputs of the corresponding PADs. An adapter card was developed [36] which was placed between the ECL outputs and the cable leading to the TDCs. When the signals pass through this 'multiplicity card', they generate a constant current of fixed duration. This current is summed up first per card and then daisy-chained from all cards of a group into the two terminating resistors of the daisy-chain cable, resulting in approximately $50 \mathrm{mV}$ per hit. Using conventional electronic modules for discriminating and coincidence decisions, it was possible to determine for a certain trigger signal, if there were enough hits detected by the straws. The corresponding logic is included in the scheme of fig. 14 . Very clean cuts were possible up to hit counts of about 15.

During the first runs using this feature, a minimum hit count of $3,4,5$, and 5 was requested from the four groups of straws if a four-prong trigger was present. With these settings, an agreement of $98 \%$ was reached with the respective off-line cuts. Allowing a disagreement by no more than one hit, e.g. due sometimes to a rather big time jitter, up to $99.8 \%$ were obtained. The accepted trigger rate was reduced by about $35 \%$.

\section{Conclusions}

We have designed, built, installed, and operated a barrel-shaped drift-tube detector of 1400 straws as a tracker in the JETSET experiment at LEAR. High-accuracy three-dimensional space information is obtained by employing both drift-time and chargedivision readout. The information is processed via specifically developed electronics.

The barrel straws were operating reliably and performed well during the 1991-1993 data-taking runs of JETSET. Work is currently in progress to obtain refined calibration and alignment parameters. The possible use of the $d E / d x$ information is at present being studied and will be discussed in a forthcoming paper [37].

\section{Acknowledgements}

During early stages of the project we have benefited from discussions with R.K. Böck, G. Bowden, J. Kirkby and F. Sauli on the principal design of the barrel straw chamber. Advice on questions of the front-end electronics has been provided by E. Chesi, T. Kondo and J.C. Santiard. For their work on prototype straws we thank P. Anzoli and D. Ploujoux. Valuable help with test-beam measurements has been given by T. Fearnley, T. Johansson, B. Mouëllic and S. Ohlsson. The high-precision end plates of the final barrel chamber have been skilfully fabricated by K. Rich. Construction and commissioning of the detector and its electronics has been speeded up with the help from W. Dutty, R. Fastner and H. Schmitt. The initial charge-division calibration for each of the 1400 barrel straws has been performed with the help of J. Kuipers. We also acknowledge 
contributions made by CERN summer students Y. Jading and O. Olsen. The work reported here has been supported partly by CERN and by the German Bundesministerium für Forschung und Technologie.

\section{References}

[1] For a review see: W.H. Toki, preprint SLAC-PUB-5232 (1990).

[2] P. Baringer et al., Nucl. Instrum. Methods A254 (1987) 542.

[3] W.W. Ash et al., Nucl. Instrum. Methods A261 (1987) 399;

H.N. Nelson, report SLAC-322 (1987).

[4] M.T. Ronan et al., IEEE Trans. Nucl. Sci. 35 (1988) 329;

TPC Collaboration, internal report TPC-LBL-85-20 (1985).

[5] W.T. Ford et al., Nucl. Instrum. Methods A255 (1987) 486.

[6] J. Adler et al., Nucl. Instrum. Methods A276 (1989) 42.

[7] V.M. Aulchenko et al., Nucl. Instrum. Methods A283 (1989) 528.

[8] M. Frautschi et al., Nucl. Instrum. Methods A307 (1991) 52.

[9] C. Biino et al., Nucl. Instrum. Methods A271 (1988) 417

and IEEE Trans. Nucl. Sci. 36 (1989) 98.

[10] S. Bhadra et al., Nucl. Instrum. Methods A268 (1988) 92.

[11] See, for example: F. Abe et al., report KEK Internal 88-1 (1988).

[12] See, for example: D.G. Cassel et al., preprint SLAC-Pub-4130 (1986);

R. DeSalvo, Cornell preprint CLNS 87/52 (1987);

ATLAS Collaboration, Letter of Intent CERN/LHCC/92-4/I2 (1993);

L3P Collaboration, Letter of Intent CERN/LHCC/92-5/I3 (1993).

[13] N. Hamann et al., Proc. International School on Physics with Low-Energy Antiprotons (4th Course: Medium-Energy Antiprotons and the Quark-Gluon Structure of Hadrons), Erice, 1990, eds. R. Landua, J.-M. Richard and R. Klapisch (Plenum Press, New York, 1991), p. 165.

[14] L. Bertolotto et al., Nucl. Phys. A558 (1993) 27c.

[15] G. Botta, Tesi di Laurea, Universitá di Genova (1991).

[16] R. Jones et al., Nucl. Instrum. Methods A323 (1992) 386.

[17] T. Sefzick, Diplomarbeit, Universität Bonn (1988), Jül-Spez 480, Forschungszentrum Jülich (1988);

A. Empl, Diplomarbeit, Universität Bonn (1989), Jül-Spez 510, Forschungszentrum Jülich (1989);

J. Thimmel, Diplomarbeit, Universität Bonn (1993), Jül 2804, Forschungszentrum Jülich (1993).

[18] D.W. Hertzog et al., Nucl. Instrum. Methods A294 (1990) 446.

[19] T. Johansson et al., private communication.

[20] The extruded tubes were made of the ISO $6061 \mathrm{Al}-\mathrm{Si}-\mathrm{Mg}-\mathrm{Mn}$ alloy (trade name "Anticorodal') and were obtained from LN Industries SA, CH-1422 Grandson, Switzerland.

[21] Various types of glue and chemical material were obtained from Lepercq, F-69684 Chassieu, France.

[22] The end plugs of the straws as well as the rivets for fixation of the anode wires were fabricated by Gonthier, CH-1450 Sainte-Croix, Switzerland.

[23] The high-quality stainless-steel wire was obtained from Microfil Industries SA, CH-1020 Renens, Switzerland.

[24] R. Bouclier et al., Nucl. Instrum. Methods A283 (1989) 509. 
[25] The miniature coaxial cable HF 75 VMTX was obtained from Filotex, F91210 Draveil, France.

[26] N. Hamann, G. Braun and H.-J. Urban, Nucl. Instr. and Meth. A 355 (1993) 255.

[27] H.-J. Urban, J. Dittmayer and N. Hamann, User Manual for Subsystems 16-Channel Preamplifier FPF-228B and 16-Channel Postamplifier/Discriminator FPF-227B, Universität Freiburg (1991). The detailed schematics are available from the authors upon request.

[28] The four-channel charge-sensitive preamplifier chips MB43468 of Fujitsu were obtained from Hemar AG, CH-5400 Baden, Switzerland.

See also: F. Suekane, T. Kondo and S. Odaka, IEEE Trans. Nucl. Sci. 33 (1986) 73.

[29] The four-channel high-speed voltage comparators MVL407 were obtained from LeCroy SA, CH-1217 Meyrin 1, Switzerland.

[30] H. Zipse, Diplomarbeit, Universität Freiburg (1990);

M. Baumgartner, Diplomarbeit, Universität Freiburg (1992);

M. Tscheulin, Diplomarbeit, Universität Freiburg (1993).

[31] The Brooks mass-flow regulators 5850 TR and control unit 5878 were obtained from Serv' Instrumentation, F-69540 Irigny, France.

[32] J. Alberi and V. Radeka, IEEE Trans. Nucl. Sci. NS-23 (1976) 251;

F. Bird et al., IEEE Trans. Nucl. Sci. NS-33 (1986) 261;

H. Fanet and J.C. Lugol, Nucl. Instrum. Methods A301 (1991) 295.

[33] The Test Pulser FPF-249B was designed and built by G. Braun, Universität Freiburg.

[34] The 80-fold Analog Fan-Out FPF-247B was designed and built by H.-J. Urban, Universität Freiburg.

[35] The Amplitude and Time-Noise Generator FPF-102B was designed and built by G. Braun, Universität Freiburg.

[36] The ECL Multiplicity Card was designed and built by C. Bourret, R. Jones, CERN, and H.-J. Urban, Universität Freiburg.

[37] H. Wirth et al., Particle Identification and Energy Loss Measurements with the JETSET Barrel Straw Chamber, in preparation. 


\section{Figure captions}

Figure 1: Plan view of the JETSET experimental apparatus at LEAR. The elements are: 1: jet-target source; 2: jet-target sink; 3: inner trigger scintillators; 4: barrel and forward 'straw' drift-tube chambers; 5: silicon-pad detectors; 6: ring-imaging Cherenkov counters; 7: threshold Cherenkov detectors; 8: outer trigger scintillators; 9: electromagnetic calorimeter; 10: $\gamma$-veto counters.

Figure 2: End view of the complete barrel straw chamber.

Figure 3: Side view of the barrel straw inserts.

Figure 4: Photograph of a glued sub-block partially equipped with end-plug inner rings.

Figure 5: Detailed view of a part of the end plate, showing the triangular posts and the $6 \mathrm{~mm}$ holes.

Figure 6: Cross-section of an end plug assembly with the straw mounted on the end plate.

Figure 7: Photograph of an end plate partially equipped with straws.

Figure 8: Photograph of a complete half of the barrel straw chamber.

Figure 9: Block diagram for the processing of signals from the barrel straw chamber. For one pair of jumpered straws, the diagram shows the principle of front-end electronics and cabling from the chamber to the charge and time digitizers.

Figure 10: Electronic connection scheme of a wire pair as used for the charge-division read-out.

Figure 11: Calibration curve $r(t)$ as obtained after iterative corrections and starting drifttime as a function at the radius from homogeneous irradiation.

Figure 12: Radial resolution of the barrel straws as extracted from the final residual distributions as a function of the drift-time radius.

Figure 13: Longitudinal resolution of the barrel straws as a function of the $z$ coordinate.

Figure 14: Trigger logic for the readout of the straws with first- and second-level trigger and multiplicity logic. 